\title{
Electrolyzed Saline Irrigation for Elimination of Bacterial Colonization in the Empyema Space
}

\author{
Kembu Nakamoto, Motohiro Takeshige, Toshiyuki Fujii, Hiroshi Hashiyada, \\ Kazuya Yoshida, and Sadahiro Kawamoto
}

\begin{abstract}
Background: The empyema space is refractory to elimination of bacterial colonization. Electrolyzed saline (ES) was used as intra-pleural irrigation for rapid disinfection of the empyema space.

Patients and Methods: Twenty consecutive patients with para-pneumonic empyema were reviewed in this study from 2007 to 2015. The empyema space was irrigated by miniaturized thoracoscopic surgery (mini VATS), and the efficacy and safety of the use of ES were evaluated.

Results: Sixteen patients were male and four were female, with a mean age of $66.5 \pm 9.5$ y (27-90y). Bacterial cultures of the purulent effusions from all 20 patients had positive results. Seventeen patients subsequently underwent continuous catheter irrigation and drainage. No patient had chest pain during ES irrigation. Fever duration after mini VATS was $2.8 \pm 1.8 \mathrm{~d}(0-5 \mathrm{~d})$. Catheter indwelling time was $10.3 \pm 7.2 \mathrm{~d}(3-33 \mathrm{~d})$. Inflammatory markers significantly improved $(\mathrm{p}<0.05)$ within a week after mini VATS. Eighteen patients achieved pleural disinfection, and two patients had residual pathogens, one of whom later died of lung abscess. Space closure was successful in 14 patients. No patient underwent subsequent open drainage. Nineteen (95\%) patients were discharged from the hospital. This treatment was successful in $18(90 \%)$ patients, and one (5.6\%) patient experienced recurrence.
\end{abstract}

Conclusions: The ES irrigation facilitates the rapid disinfection and closure of the empyema space.

$\mathbf{I}$ N FIBROPURULENT EMPYEMA, current conservative management with intravenous antibiotics and pleural drainage frequently fails $[1,2]$. The key to treating patients with empyema is the cleansing and closure of the empyema space for relief of septic conditions, facilitation of pleural epithelialization, and reduction of bacterial colonization [2-4]. The current strategy for achieving this frequently necessitates invasive surgical approaches with long hospital stays [2-5]. Thus, there is a need for a novel approach to resolve these problems [3].

Electrolyzed saline (ES) is listed in the guidelines of the Centers for Disease Control and Prevention (CDC) as a new irrigating and disinfectant agent with non-toxic actions on biologic tissues and broad-spectrum bactericidal activity [6-8]. The ES was used for intra-pleural irrigation by videoassisted thoracoscopic surgery with a miniaturized thoracoscope under local anesthesia (mini VATS), which we primarily used as an intra-thoracic diagnostic alternative to exploratory thoracotomy [9]. In the present study, we investigated the efficacy and safety of ES irrigation with mini VATS as a radical and less invasive treatment of patients with acute empyema thoracis.

\section{Patients and Methods}

The Institutional Review Board of our hospital granted ethical approval for this study (IRB \#2602, SMH, March 24, 2015). Individual patient consent was obtained.

\section{Patients}

Twenty consecutive patients with para-pneumonic empyema in the fibropurulent phase, referred by respiratory physicians after the failure of initial treatment with or without pleural drainage, were enrolled in this retrospective study between October 2007 and September 2015. The mean patient age was $66.5 \pm 9.5 \mathrm{y}(27-90 \mathrm{y})$. Of the 20 patients, 16 were male, and 16 patients had co-morbidities with a Charlson Comorbidity Index score [10] of more than one point.

Division of General Thoracic Surgery, Department of Surgery, Division of Respiratory Medicine, Infection Control Team, Shunan Memorial Hospital, Kudamatsu, Yamaguchi, Japan.

(C) Kembu Nakamoto, et al., 2016; Published by Mary Ann Liebert, Inc. This Open Access article is distributed under the terms of the Creative Commons Attribution Noncommercial License (http://creativecommons.org/licenses/by-nc/4.0/) which permits any noncommercial use, distribution, and reproduction in any medium, provided the original author(s) and the source are credited. 
Eight patients had chronic pulmonary disease, and seven patients had a diagnosis of immunosuppression with severe diabetes mellitus, had steroid-dependent connective tissue disease, or were post-pneumonectomy for lung cancer. Four patients had senile dementia, depression, or schizophrenia. Only four patients had a comorbidity score of zero points. Pathogens identified were Streptococcus strains, Peptostreptococcus strains, Fusobacterium strains, Klebsiella pneumoniae, methicillin-resistant Staphylococcus aureus (MRSA), Haemophilus influenzae, Pseudomonas aeruginosa, Escherichia coli, and one Aspergillus strain. Most of the pathogens were oral microflora (Table 1).

\section{Follow-up}

Empyema space follow-up examinations included computed tomography (CT), ultrasonography, or thoracentesis. The outcomes of the treatment strategy were evaluated using the following tests: Bacterial culture; presence of the hematologic inflammatory marker, C-reactive protein (CRP); leukocyte and neutrophil counts on the day of admission, just before mini VATS, post-mini VATS days five to seven, and just before hospital discharge; treatment period; duration of hospital stay after mini VATS; recurrence of empyema; and incidence of adverse events, such as chest pain during ES irrigation. The end points of evaluation were disinfection of the empyema space and hospital discharge to home or a nursing home.

\section{Preparation of ES}

The ES was produced from a $0.1 \%$ salt and tap water mixture, with a final salt concentration lower than that of physiologic saline, using a water electrolysis generator (Oxilyzer Medical C-L, Koken Ltd., Tokyo). Electrolyzed acidic water with a $\mathrm{pH}$ of less than 2.7 was generated in the anode compartment and was collected for use in irrigation. Sterilization was unnecessary in the preparation of ES.

\section{Bactericidal activity of custom-made ES}

The minimum bactericidal concentration (MBC) of the ES against MRSA, Enterococcus faecalis, Bacillus cereus, $P$. aeruginosa, E. coli, a Streptococcus strain, Candida albicans,

Table 1. Patient Characteristics AND EMPYEMa Pathogens

Patient characteristics

\begin{tabular}{lc}
\hline Number of patients & 20 \\
Age (years old) & $66.5 \pm 9.5(27-90)$ \\
Gender: male/female & $16 / 4$ \\
Charlson Comorbidity Score & $2.0 \pm 1.2(0-4)$ \\
\hline
\end{tabular}

Pathogens*

Streptococcus strain

Peptostreptococcus strain

Fusobacterium strain

Klebsiella pneumoniae

Methicillin-resistant Staphylococcus aureus

Pseudomonas aeruginosa

Haemophilus influenzae

Escherichia coli

Aspergillus strain

8

3

3

2

2

2

1

1

*Overlapping description. and a Aspergillus strain was examined according to the method of von Beck et al. [11] and was compared with $80 \%$ ethanol, $0.05 \%$ chlorhexidine, $10 \%$ povidone iodine, and tap water. Test samples of $50 \mathrm{mcL}$ of broth, in which $1.5-3 \times 10^{8}$ colony-forming units/mL of bacteria were suspended, were mixed with $5 \mathrm{~mL}$ of ES, serially diluted with tap water or control disinfectants, for $1 \mathrm{~min}$. Then $50 \mathrm{mcL}$ of each mixture was added to $5 \mathrm{~mL}$ of broth and incubated for $72 \mathrm{~h}$ at $37^{\circ} \mathrm{C}$, after neutralizing the bactericidal activity.

\section{Indication for procedures}

The indication for ES irrigation with mini VATS was as follows: Unstable clinical features and inflammatory markers after initial management with intravenous antibiotics with or without pleural drainage, macroscopic purulent or bacteriapositive effusions, or multiple septations with fibrin debris on chest CT or ultrasonography.

\section{Mini VATS procedure}

The detailed procedure of the mini VATS was described previously [9]. Briefly, patients were placed in a lateral position and were restrained on a mattress (Magic Bed, Okada Medical Co. Ltd., Tokyo) on the operating table. A thoracoport incision, for the removal of fibrin debris, purulent effusion, ES topical irrigation, and monitoring using a minithoracoscope (2.9 mm Ideal Eyes, Stryker Co., Kalamazoo, $\mathrm{MI}$ ), was made at an appropriate site, determined by ultrasonography. If the thoracoscope monitoring disturbed the handling of other operating tools, an additional incision was made using a needle port (2/3 mm Endopath Bladeless Trocar, Johnson \& Johnson, New Brunswick, NJ) for the minithoracoscope.

Fibrin debris was removed to form a single lumen, and the pleural cavity was washed out and cleaned by topical irrigation with 2-3 L of warm ES. At the end of the procedure, a double-lumen silicon catheter (18F Phicon Samp Catheter, Fuji Systems Co., Tokyo), for continuous ES irrigation and pleural drainage with low-pressure suction, was inserted into the dead space cavity until the clinical features improved and the drained fluid became clear and negative for bacteria.

The bactericidal activity of the ES was promptly deactivated by protein substances; the continuous ES irrigation was our routine procedure for keeping the activity level. The ES was set to drip continuously at a rate of $60-80 \mathrm{~mL} / \mathrm{h}$ into the inflow of the double-lumen catheter.

\section{Statistical analysis}

The data are expressed as mean \pm standard deviation. Microsoft Excel was used for the statistical analysis, and a $\mathrm{p}<0.05$, by paired $t$-test with two-tailed distribution, was defined as significant.

\section{Results}

\section{The $M B C$ of $E S$}

The ES diluted with tap water to $50 \%$ completely inhibited the growth of microbes, except for the spore-forming bacteria, Bacillus cereus, for $72 \mathrm{~h}$; however, an ES diluted to $10 \%$ and the control, tap water, were positive for bacterial growth within $24 \mathrm{~h}$. Thus, the MBC of our custom-made ES was $50 \%$. The bactericidal activity of this solution was similar to 
Table 2. Minimum Bactericidal Concentration of Custom-Made Electrolyzed Saline

\begin{tabular}{|c|c|c|c|c|c|c|c|c|}
\hline \multirow[b]{2}{*}{ Pathogen } & \multicolumn{4}{|c|}{ Dilution ratio (ES/tap water) } & \multicolumn{4}{|c|}{ Control } \\
\hline & Original & $1 / 1$ & $1 / 4$ & $1 / 9$ & Tap water & Ethanol $^{1}$ & Chlorhexidine $^{2}$ & $P I^{3}$ \\
\hline \multicolumn{9}{|l|}{ Growth of microbes } \\
\hline MRSA & - & - & - & + & + & - & + & - \\
\hline Enterococcus faecalis & - & - & - & + & + & - & + & + \\
\hline Bacillus cereus & + & + & + & + & + & + & + & + \\
\hline P. aeruginosa & - & - & - & + & + & - & + & - \\
\hline Escherichia coli & - & - & - & + & + & - & + & - \\
\hline Streptococcus strain & - & - & + & + & + & - & - & - \\
\hline Candida albicans & - & - & + & + & + & - & + & - \\
\hline Aspergillus strain & - & - & + & + & + & - & + & + \\
\hline
\end{tabular}

$\mathrm{ES}=$ electrolyzed saline, MRSA = methicillin-resistant Staphylococcus aureus, $P=$ Pseudomonas.

${ }^{1} 80 \%$ ethanol; ${ }^{2} 0.05 \%$ chlorhexidine; ${ }^{3} 10 \%$ povidone iodine.

$80 \%$ ethanol and superior to $0.05 \%$ chlorhexidine and $10 \%$ povidone iodine (Table 2).

\section{Procedures and clinical features}

In three patients with mental disturbance, an indwelling catheter was not used for continuous irrigation and drainage during the mini VATS procedure. Four patients received repeated mini VATS procedures for persistent purulent exudate because of the incomplete removal of fibrin debris from the residual empyema pockets. None of the patients received general anesthesia. The average duration of the mini VATS procedure was $43 \pm 14 \mathrm{~min}(20-80 \mathrm{~min})$. The average duration of continuous irrigation until the removal of the doublelumen catheter was $10.3 \pm 7.2 \mathrm{~d}(3-33 \mathrm{~d})$ in the 17 patients with indwelling catheters.

The duration of parenteral antibiotics for the initial treatment was $9.1 \pm 8.3 \mathrm{~d}$ and after mini VATS was $12.4 \pm 11.3 \mathrm{~d}$. The duration of fever above $37.0^{\circ} \mathrm{C}$ after ES irrigation was $2.8 \pm 1.8 \mathrm{~d}$ with prompt improvement in other clinical features, and before mini VATS was $8.6 \pm 7.4 \mathrm{~d}$. Fever subsided within $1-5 \mathrm{~d}$ in the three patients without indwelling catheters. Three patients did not have fever but experienced malaise throughout the treatment period. No adverse effects, such as chest pain or abnormal hematologic examination results, were observed during ES irrigation (Table 3).

\section{Changes in inflammatory markers}

The hematologic inflammatory marker, CRP, and leukocyte and neutrophil counts improved with intravenous anti- biotics, but the levels of these markers were unstable until mini VATS was performed. Intervention using ES irrigation with mini VATS significantly improved these markers, which returned to stable levels within $7 \mathrm{~d}$ (Table 4).

\section{Outcomes of ES irrigation with mini VATS}

Eighteen of the 20 patients recovered from empyema, with negative results of bacterial cultures of pleural fluid. A residual presence of pathogens with decreased dead space accompanying occasional purulent discharge without other clinical features was observed during an outpatient clinic visit in one patient, in whom the indwelling catheter was accidentally removed before disinfection of the pleural effusion. One patient, with a lung abscess and pleural air leakage, had persistent bacterial colonization.

Complete closure of the pleural dead space was observed in all but six patients (Fig. 1). One of the six patients with a residual space had a post-pneumonectomy space; the pleural surface of this space was covered with healthy granulation, and the fluid of the space was replaced with clear serous effusion after the second mini VATS procedure or follow-up thoracentesis. There was one hospital death of a patient with a lung abscess.

Nineteen patients were discharged from our hospital, either to their homes or nursing homes. The average duration of hospital stay after mini VATS was $23.9 \pm 11.7 \mathrm{~d}$. None of the 20 patients underwent subsequent open thoracostomy. One of the 18 patients treated successfully had a recurrence of empyema in the residual space 2 mo later, with pneumonia.

Table 3. Electrolyzed Saline Irrigation Procedures and Clinical Features

\section{Procedures and clinical features}

$\begin{aligned} & 3(1) \\ & 17(3) \\ & 43 \pm 14 \quad(20-80) \\ & 10.3 \pm 7.2(3-33: \mathrm{n}=17) \\ & 9.1 \pm 8.3(1-60) / 12.4 \pm 11.3(0-37)^{\mathrm{a}} \\ & 8.6 \pm 7.4(0-60) / 2.8 \pm 1.8(0-10)^{\mathrm{a}} \\ & \text { None }\end{aligned}$

rigation procedure (number of patients)

Mini VATS (repeated)

Mini VATS with catheter indwelling (repeated)

Operative time for mini VATS (min)

Catheter indwelling period after mini VATS (d)

Antibiotic period before/after mini VATS (d)

Febrile period before/after mini VATS (d)

Chest pain during irrigation

Mini VATS = video-assisted thoracoscopic surgery with miniaturized scopes under local anesthesia.

${ }^{\mathrm{a}}$ Not significant. 
Table 4. Transition of Hematologic Inflammatory Markers

\begin{tabular}{|c|c|c|c|c|c|c|c|}
\hline Marker \date & Admission & & Pre-mini VATS & & Post-mini VATS & & Discharge \\
\hline CRP (mg/dL) & $17.0 \pm 7.1$ & $\leftarrow \mathrm{NS} \rightarrow$ & $13.2 \pm 5.7$ & $\leftarrow \mathrm{p}<0$ & 5.6 & $\leftarrow \mathrm{NS} \rightarrow$ & 2.6 \\
\hline WBC $\left(10^{3} / \mathrm{mcL}\right)$ & $16.5 \pm 8.1$ & $\leftarrow \mathrm{NS} \rightarrow$ & $13.1 \pm 4.3$ & $\leftarrow \mathrm{p}=0.04 \rightarrow$ & $7.7 \pm 2.5$ & $\leftarrow \mathrm{NS} \rightarrow$ & $7.2 \pm 2.4$ \\
\hline Neut $\left(10^{3} / \mathrm{mcL}\right)$ & $14.4 \pm 7.8$ & $\leftarrow \mathrm{NS} \rightarrow$ & $10.0 \pm 3.3$ & $\leftarrow \mathrm{p}<0.001 \rightarrow$ & $5.8 \pm 2.3$ & $\leftarrow \mathrm{NS} \rightarrow$ & $4.9 \pm 3.1$ \\
\hline
\end{tabular}

Mini VATS = video-assisted thoracoscopic surgery with miniaturized scopes under local anesthesia; $\mathrm{CRP}=\mathrm{C}$-reactive protein; NS $=$ not significant; $\mathrm{WBC}=$ white blood cell; Neut $=$ neutrophil.
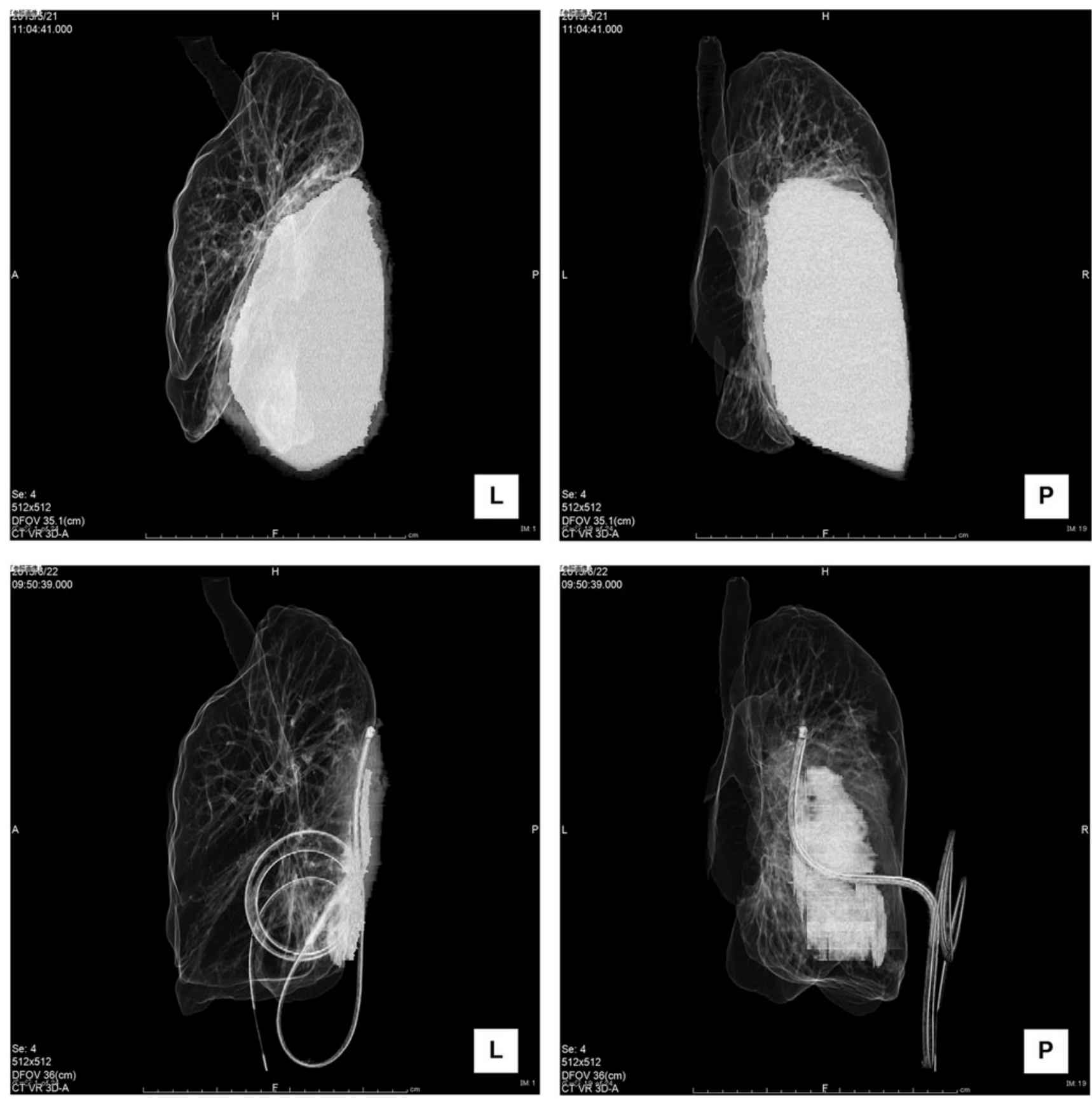

FIG. 1. Rapid disinfection and uniform closure of the empyema space by intra-pleural irrigation with electrolyzed saline (volume-rendered three-dimensional computed tomography images of the empyema space). Top: Medial and posterior views of a 66-year-old male with encapsulated massive purulent effusion on the right side before electrolyzed saline irrigation. Bottom: Medial and posterior views of uniform and rapid closure of the empyema space after removal of fibrin debris under mini-VATS and continuous irrigation with electrolyzed saline through an indwelled catheter. MiniVATS $=$ video-assisted thoracoscopic surgery with miniaturized scopes under local anesthesia. 
Table 5. Outcomes of Empyema by Electrolyzed Saline Irrigation Under Mini VATS

\begin{tabular}{lc}
\hline Outcomes & \\
\hline Residual pathogens: No/yes & $18 / 2$ \\
Pleural dead space: Closed/residual & $14 / 6$ \\
Hospital discharge/hospital death & $19 / 1$ \\
Hospital stay after mini VATS (d) & $23.9 \pm 11.7(7-40)$ \\
Curability: Succeeded (recurred)/failed & $18 \quad(1) / 2$ \\
\hline
\end{tabular}

Mini VATS $=$ video-assisted thoracoscopic surgery with miniaturized scopes under local anesthesia.

Thus, the curative rate of empyema using ES irrigation with mini VATS was $90 \%$, and the recurrence rate was $5.6 \%$ (Table 5).

\section{Discussion}

The key treatment of patients with empyema includes the cleansing and closure of the pleural cavity to reduce bacterial colonization [2-4]. Residual microbes and multiple loculations with massive purulent fibrin debris may interfere with this treatment, and conservative treatment with intravenous antibiotics and catheter drainage is frequently replaced by an invasive procedure with long treatment periods. Thus, a novel, effective, and less invasive approach was required to resolve these problems [3].

The use of ES became widespread in Japan for medical environmental disinfection when MRSA became a focus of hospital infections in the 1990s [7,8]. The ES is effective against a broad spectrum of pathogens, including spores, fungi, viruses, acid-fast bacilli, and MRSA [7]. The bactericidal activity of ES is similar to that of $80 \%$ ethanol and superior to those of chlorhexidine and povidone iodine [12].

In the present study, the bactericidal activity of our ES was similar to that of ethanol against a broad spectrum of pathogens, except spore-forming bacteria. The ES is immediately converted to water after contact with an organism and is not corrosive to skin, mucous membranes, or organic materials [8]. The ES tastes and smells of chlorine, slightly more strongly than municipal tap water. The ES is used primarily for rinsing fresh vegetables or seafood, medical tool disinfection, hand cleansing, oral rinsing, and in dermatology and dentistry $[13,14]$.

Its usefulness was further extended in Japan after several studies used ES to manage mediastinitis after cardiac operations and peritoneal abscess after abdominal operations $[15,16]$. The ES has been found to promote re-epithelialization, increasing the proliferation of lymphocytes and macrophages associated with collagen deposition for surgical incision healing [14]. Further, ES was listed in the $2008 \mathrm{CDC}$ guidelines as a new irrigation and disinfectant agent with non-toxic action on biological tissues [6].

Since 1996 at our hospital, we have used ES primarily for brush cleansing of fenestrated cavities of post-operative empyema with bronchofistula. The time needed for surgical incision disinfection and healthy epithelialization of the pleural surface was significantly shortened in comparison with that of conventional open-packing with sterilized gauze. We have also developed another successful approach for the management of descending necrotizing mediastinitis using
ES irrigation through an indwelling catheter with mediastinoscopy (not published). Thus, based on these findings, we used ES for closed-space irrigation of acute empyema without bronchofistula.

Removal of fibrin debris with mini VATS facilitated uniform irrigation and drainage of the empyema space. The ES was used for rapid disinfection of the pleural membranes instead of removal by decortication. Continuous ES irrigation with low-pressure suction promoted rapid disinfection and epithelialization of the purulent space and was followed by spontaneous closure of the dead space. Single topical ES irrigation may be effective even when continuous indwelling catheter use should be avoided.

The principal goal of empyema treatment is closure of the dead space $[2,3]$. Findings from our patient with an infected post-pneumonectomy space suggest, however, that the closure of the residual space may be unnecessary if disinfection of the pleura is achieved. The patient demonstrated clear epithelialization of the pleural surface on the second mini VATS, and there were negative results for microbes in clear fluid on follow-up thoracentesis. No adverse events, such as chest pain or hematologic abnormalities, occurred during ES irrigation. In addition, we achieved a $90 \%$ curative rate of empyema with a mean hospital stay of $23.9 \mathrm{~d}$, including comorbidity treatment and post-medication rehabilitation, and a $5.6 \%$ recurrence rate.

Our previous study on saline irrigation for empyema had a $67 \%$ disease cure rate after $45 \mathrm{~d}$ of treatment. Long treatment periods were required with physiologic saline to achieve a complete cure (not published). Our disease cure rate results are superior or comparable to those of surgical interventions reported in previous studies [3-5,12,17], suggesting that this approach could replace aggressive surgical approaches for the management of acute empyema thoracis. Pneumothorax, which is a sign of bronchopleural fistula, may be the main cause of treatment failure in the patient with lung abscesses. In cases of severe bronchofistula, we elect to perform thoracostomy, a conventional surgical procedure, to prevent the aspiration of ES into the contralateral lung.

Several Japanese companies invented the electrolysis generators meeting Japanese national policies for food and environmental sanitation in response to mass food poisonings of $E$. coli O-157 in the mid-1990s. The ES generated by the electrolysis of a weak saline has a strong broad-spectrum bactericidal action in which hypochlorite is considered the main disinfectant [6]. The electrolysis generators for ES are commercially available $[18,19]$.

The ES has no adverse effects on the human body [8,13,14], or the environment, because it is immediately converted to water after contact with an organism. A sterilization process is unnecessary for ES preparations, because ES, itself, is the sterilizer of routinely washed and dried medical instruments. The cost of producing ES is quite low: Approximately US $\$ 0.25$ for $50 \mathrm{~L} / \mathrm{d}[18]$.

Closed space irrigation with ES may be a potentially useful strategy for management of refractory bacterial colonization of other infected dead spaces, as an alternative to invasive open drainage. We now use ES as a standard wound disinfectant instead of conventional disinfectants such as ethanol, chlorhexidine, and povidone iodine.

The double-lumen silicone indwelling catheter is our preferred catheter for use in the pleural cavity against 
hydropneumothorax. It has the appropriate softness, flexibility, and rigidity for manual insertion and looping in the pleural cavity with effective drainage. It can be inserted into a small pleural dead space without the catheter collapsing. This double-lumen catheter is convenient for both irrigation and drainage of the empyema cavity for long periods.

The most common causative disease in our study was considered to be aspiration pneumonia, because the major pathogens were oral microflora. Aspiration pneumonia is a common disease in the field of geriatric medicine. Such patients may have para-pneumonic effusions, leading progressively to life-threatening empyema thoracis $[1,2]$. In terms of minimally invasive strategies, intervention using ES irrigation with mini VATS could contribute to the treatment of patients with poor performance status.

\section{Statistical limitations}

Our study is a preliminary report of ES, and a randomized control study is required for clearing the effects of ES alone. Thus, further investigation of this minimally invasive and radical approach for treatment of patients with empyema is warranted, using larger cohorts.

\section{Conclusion}

ES irrigation with mini VATS for acute empyema is an effective, minimally invasive, simple, and inexpensive approach for rapid disinfection and healing of the pleural cavity.

\section{Acknowledgments}

We thank Takashi Yamanaka, PhC, and Kazuo Nagano, MT, for their assistance with electrolyzed saline preparation and microbiologic examinations.

\section{Author Disclosure Statement}

No competing financial interests exist.

\section{References}

1. Rahman NM, Kahan BC, Miller RF, et al. A clinical score (RAPID) to identify those at risk for poor outcome at presentation in patients with pleural infection. Chest 2014; 145:848-855.

2. Davies CW, Gleeson FV, Davies RJ; BTS Pleural Group. BTS guidelines for the management of pleural infection. Thorax 2003;58:ii18-ii28.

3. Molnar TF. Current surgical treatment of thoracic empyema in adults. Eur J Cardiothorac Surg 2007;32:422-430.

4. Mandal AK, Thadepalli H, Mandal AK, Chettipally U. Outcome of primary empyema thoracis: Therapoitic and microbiologic aspects. Ann Thorac Surg 1998;66:17821786.

5. Wait MA, Beckles DL, Paul M, et al. Thoracoscopic management of empyema thoracis. J Minim Access Surg 2007;3:141-148.
6. Rutala WA, Weber DJ; The Healthcare Infection Control Practices Advisory Committee (HICPAC). Guideline for disinfection and sterilization in healthcare facilities, 2008. www.cdc.gov/hicpac/Disinfection_Sterilization/acknowledg .html (Last accessed Feb 1, 2015).

7. Kiura H, Sano K, Morimatsu S, et al. Bactericidal activity of electrolyzed acid water from solution containing sodium chloride at low concentration, in comparison with that at high concentration. J Microbiol Methods 2002;49:285-293.

8. Rutala WA, Weber DJ. New disinfection and sterilization methods. Emerg Infect Dis 2001;7:348-353.

9. Nakamoto K, Maeda M, Okamoto T, et al. Preoperative diagnosis with video-assisted thoracoscopy with miniaturized endoscopes in general thoracic surgery: A preliminary study. Chest 1998;114:1749-1755.

10. Charlson ME, Pompei P, Ales KL, MacKenzie CR. A new method of classifying prognostic comorbidity in longitudinal studies: Development and validation. J Chronic Dis 1987;40:373-383.

11. Von Beck EG, Borneff J, Grun L, et al. Empfehlungen fur die Prufung und Bewertung der Wirksamkeit chemischer Desinfektionsverfahren. Int J Med Microbiol 1977;165: 335-380.

12. Tanaka H, Hirakata Y, Kaku M, et al. Antimicrobial activity of superoxidized water. J Hosp Infect 1996;34:43-49.

13. Huang Y, Hung Y, Hsu S, et al. Application of electrolyzed water in the food industry. Food Control 2008;19:329-345.

14. Thorn RM, Lee SW, Robinson GM, et al. Electrochemically activated solutions: Evidence for antimicrobial efficacy and applications in healthcare environments. Eur J Clin Microbiol Infect Dis 2012;31:641-653.

15. Hayashi H, Kumon K, Yahagi N, et al. Successful treatment of mediastinitis after cardiovascular surgery using electrolyzed strong acid aqueous solution. Artif Organs 1997;21:39-42.

16. Inoue Y, Endo S, Kondo K, et al. Trial of electrolyzed strong acid aqueous solution lavage in the treatment of peritonitis and intraperitoneal abscess. Artif Organs 1997; 21:28-31.

17. Yamaguchi M, Takeo S, Suemitsu R, et al. Video-assisted thoracic surgery for fibropurulent thoracic empyena: A bridge to open thoracic surgery. Ann Thorac Cardiovasc Surg 2009;15:368-372.

18. Strongly acidic "Denkaisui" electrolyzed water generator. www.koken-ltd.co.jp/english/ktech_sadewg.htm (Last accessed February 1, 2015).

19. EOwater Ltd. Killing bacteria: Quicker, safer, cheaper. www.eowater.ltd.uk/about-rox.html (Last accessed June 6, 2016).

Address correspondence to: Dr. Kembu Nakamoto

Division of General Thoracic Surgery Shunan Memorial Hospital 1-10-1 Ikunoya-minami, Kudamatsu Yamaguchi 744-0033 Japan

E-mail: nakamoto@hcsdojinkai.or.jp 